\title{
Genetic variation of inbreeding depression among floral and fitness traits in Silene nutans
}

\author{
J Thiele $^{1}$, T Hansen ${ }^{2}$, HR Siegismund ${ }^{2}$ and TP Hauser ${ }^{1}$ \\ ${ }^{1}$ Department of Agriculture and Ecology, University of Copenhagen, Frederiksberg C, Denmark and ${ }^{2}$ Institute of Biology, University \\ of Copenhagen, Universitetsparken, Copenhagen $\varnothing$, Denmark
}

\begin{abstract}
The magnitude and variation of inbreeding depression (ID) within populations is important for the evolution and maintenance of mixed mating systems. We studied ID and its genetic variation in a range of floral and fitness traits in a small and large population of the perennial herb Silene nutans, using controlled pollinations in a fully factorial North Carolina II design. Floral traits and early fitness traits, that is seed mass and germination rate, were not much affected by inbreeding $(\delta<0.2)$. In contrast, 'late' fitness traits and multiplicative fitness suffered severely from inbreeding $(\delta>0.4)$. Lack of genetic correlations indicated that ID in floral, early and late traits is genetically decoupled. There was a trend that the
\end{abstract}

smaller population was less affected by ID than the large one, although the differences were not significant for most traits. Hence, evidence for purging of deleterious alleles remains inconclusive in this study. Genetic variation in ID among paternal families was statistically significant in most floral and all seed traits, but not in late fitness traits. However, some paternal families had $\delta<0.5$, even in the multiplicative fitness measure that suffered most from ID $(\delta=0.74)$, suggesting that the mixed mating system of $S$. nutans might be evolutionary stable.

Heredity (2010) 104, 52-60; doi:10.1038/hdy.2009.103; published online 19 August 2009

Keywords: breeding-system evolution; genetic correlation; North Carolina II design; population genetics; purging; quantitative genetics

\section{Introduction}

Inbreeding depression (ID) is a driver of mating-system evolution and a major concern in the management of many endangered species (for example Goodwillie et al., 2005; Charlesworth, 2006; Ouborg et al., 2006; Allendorf and Luikart, 2007). The severity and onset of ID and its ability to evolve with changes in the mating system depend on the underlying genetic basis. Among the contributing factors are the degree of dominance of loci affecting ID and the number of loci affecting the traits that suffer from ID (Charlesworth et al., 1990). Further, a population's ability to purge deleterious mutations that cause ID depends on the strength of the deleterious effects and the degree of dominance or recessiveness of the mutated alleles. If, for example, ID is caused by deleterious mutations of large effects, even populations with low-selfing rates will effectively purge the genetic load through natural selection and, hence, suffer little ID at mutation-selection equilibrium. If, on the other hand, ID is caused by many recessive and only mildly deleterious alleles, genetic load will not be purged effectively almost regardless of selfing rate (Charlesworth and Charlesworth, 1999).

In self-fertile plants, inbreeding can occur either through self-fertilization or through matings between

Correspondence: Associate Professor TP Hauser, Department of Agriculture and Ecology, University of Copenhagen, Rolighedsvej 21, 1958 Frederiksberg C, Denmark.

E-mail: tpha@life.ku.dk

Received 11 December 2008; revised 20 May 2009; accepted 26 May 2009; published online 19 August 2009 otherwise related individuals. In populations with relatively small effective population numbers, the limited array of possible mating partners will result in a build up of relatedness among individuals and in inbreeding per se (Ellstrand and Elam, 1993). Thus, comparatively small populations could suffer less ID than comparatively large populations, because of the higher inbreeding levels in the former and, hence, the more effective purging of genetic load.

One aspect of ID in natural plant populations that has received little explicit empirical attention is the degree to which families within or among populations vary in their level of ID (Fox, 2005; Kelly, 2005; Kelly and Tourtellot, 2006; Ouborg et al., 2006). A theoretical model on the evolution of mating-system modifiers showed that an association between selfing promoters and viability determining loci could build up, resulting in an invasion of selfing-promoting genes, if ID was below some threshold value and caused by recessive deleterious alleles (Uyenoyama and Waller, 1991a, b,c). However, strong variation in the ID among families could facilitate the spread of selfing promoters even in populations with high average ID (above 0.5).

Most studies on ID have focused on traits closely related to fitness. The degree to which inbreeding affects fitness traits of different life stages differs between species that are predominantly selfers and species that are predominantly outcrossers (Husband and Schemske, 1996; Ouborg et al., 2006). There is a tendency of selfing species to suffer more severe ID in late life-cycle traits compared with early traits, whereas outcrossing species tend to suffer more severe ID in early traits (for example 
Mustajärvi et al., 2005). Husband and Schemske (1996) suggested that the ID in early traits is caused by recessive lethals, which are effectively purged through inbreeding, whereas ID in late traits is caused by recessive and only mildly deleterious mutations that are very difficult to purge even in highly inbred populations.

Floral traits, such as the size of petals, anthers and ovaries, have been less studied with regard to ID, although they may be affected as well. As mechanisms of selection may be different for floral and fitness traits, it is important to know whether the magnitude of ID is different for these two sets of characters.

In this paper, we test the quantitative genetics of ID in Silene nutans L. by addressing the following questions: (1) Does the level of ID vary with population size? (2) Does ID vary between fitness and non-fitness traits expressed at different life-cycle stages? (3) To which degree is the ID observed in different traits under the same genetic control? (4) Is there genetic variation in ID among families?

\section{Materials and methods}

\section{Study species}

S. nutans is a rosette forming perennial herb of dry and open non-acidic grassland communities. It has a wide distribution range from France and Belgium in Western Europe to the Urals and the Caucasus in the East. Flowers are usually hermaphroditic, protandric and pollinated by a number of different insect species, especially nocturnal moths (Jürgens et al., 1996). Selfing rate is intermediate, but with a large variation among regions (van Rossum and Prentice, 2004), populations, individuals and flowers of the same plant (Philipp and Weidema, unpublished). Among 10 Danish populations, the selfing rate ranged between 0.08 and 0.61 and covaried negatively with population size $(r=-0.82$; $P=0.002$; Philipp and Weidema, unpublished). In Denmark, $S$. nutans is relatively rare and many populations are small and apparently isolated.

The individuals used in this experiment come from two Danish populations located $60 \mathrm{~km}$ apart on the island of Zealand. 'Børsen' is a small, isolated population (approximately 30 individuals) growing on an inland bronze age grave mound that has been covered in grassland vegetation, to which $S$. nutans belongs, since at least 500 BC (Bruun et al., 2001). Unpublished results by Philipp and Weidema show that adult plants in this population are not inbred ( $F_{i s}$ not different from zero). 'Klint' is a very extensive coastal population of $>1000$ individuals. The inbreeding level of this population has not been studied; however, another large coastal population from the same region is not inbred ( $F_{i s}$ not different from zero). Coasts are some of the most persistent habitat types in Denmark, and it is, therefore, likely that the Klint population has existed for very long time. Thus, both populations may be very old. In what follows, B $ø$ rsen will be denoted 'Small' and Klint 'Large'.

\section{Experimental design}

Crossings and selfings: From each of the two populations, 18 individual rosettes were sampled. Nine randomly chosen individuals from each set were used as pollen acceptors (hereafter 'females') and the remaining nine were used as pollen donors (hereafter 'males'). In the $P$-generation, all nine males from each population were crossed factorially onto all nine females from the same population, according to a North Carolina II design. The first-generation seeds thus included 81 fullsib and 9 paternal half-sib families from each population. Using this design, we can estimate and compare ID for the paternal families in the $P$-generation across the same set of maternal plants. We thereby avoid the inflation of variance that would result from using different sets of maternal plants for each paternal family.

Part of the seeds was used directly for a common garden experiment (see below), whereas another part was used to produce self-pollinated offspring. For that purpose, 10 seeds (outcrossed) from each of the 162 firstgeneration crosses were randomly picked and sown into $10 \mathrm{~cm}$ pots. Four weeks after germination, the number of plants per pot was randomly reduced to one. At sexual maturity, approximately two flowers per plant were selffertilized and the resulting selfed second-generation seeds were harvested. The reason for comparing outcrossed offspring from the first generation with selfed offspring from the second generation is that they are related by having the same sets of original $P$-generation fathers; the nine paternal families from each population thus include both an outcrossed and a selfed set of offspring with the same expected paternal contribution ( $P$-paternal contribution to the offspring genome is expected to be 0.5).

Common garden trial: Approximately 10 seeds from all the 162 outcrossed and 162 selfed families were sown into pots in January 1999. Four weeks after germination, surplus plants were randomly weeded out to reduce the number of plants per pot to one. In late April, all 324 plants were transplanted in a randomized design to an outdoor common garden at the experimental farm of University of Copenhagen, approximately $10 \mathrm{~km}$ west of Copenhagen. Distance between rows was $80 \mathrm{~cm}$ and among plants $25 \mathrm{~cm}$; plants were watered when necessary, but not fertilized or treated with pesticides. By the summer of 2000, all outcrossed and 132 selfed plants had survived. The number of outcrossed plants was, therefore, also reduced (to 138, to ensure that all remaining families had sufficient outcrossed and selfed plants for statistical analysis; see Equation (1)). At flowering, the plants were allowed to pollinate each other freely.

\section{Phenotypical measurements}

We recorded an array of traits covering different life stages of the plants in the common garden trial. In this study, we discern 'fitness traits', that are related to growth and seeds and usually considered to represent plant fitness, from 'floral traits' that are related to flowering organs and usually considered to be nonfitness traits. According to the plant life cycle, we separated fitness traits into 'early' ones, that is related to seeds, and 'late' ones related to the adult phase of the plants.

Floral traits: The two populations exhibit significantly different onset of flowering (Hauser and Weidema, 2000). Therefore, we sampled the flowers over a course of 10 days in May and June 2000 to reduce variation caused by 
phenological differences in sex allocation. The plants were quite large and carried close to 1000 flowers on average. We harvested four flowers from each individual plant and kept them at $-18^{\circ} \mathrm{C}$ until dissection. The flowers were chosen at the stage in which the petals emerged from the sepals though the flowers were not 'opened' yet. This stage is easy to recognize and short $(<8 \mathrm{~h})$. We dissected flowers into sepals, petals, stamens and ovary (with style and stigma). Then the flower components were dried at $70^{\circ} \mathrm{C}$ for $36 \mathrm{~h}$ and we measured the dry weight to the nearest $10^{-6} \mathrm{~g}$.

Early fitness traits: Seed mass 1: From each of the original 162 outcrossed and 162 selfed families, we weighed 25 randomly picked seeds to the nearest $10^{-6} \mathrm{~g}$ and calculated average seed mass. Germination rate 1: From each of these crossings and selfings, 25 randomly picked seeds were sown onto wet filter paper in Petri dishes. After 20 days, we counted the number of germinated seeds and calculated the germination rate. During this period, the filter paper was kept wet. Seed mass 2: We harvested seeds from the outcrossed and selfed plants grown in the common garden trial and randomly picked fifty seeds from each plant. Then, we weighed the seeds to the nearest $10^{-6} \mathrm{~g}$ and calculated average seed mass. Germination rate 2: From each of these harvested plants, we put fifty randomly chosen seeds onto wet filter paper in Petri dishes. After 18 days, we counted the number of germinated seeds and calculated the germination rate. It is important to appreciate the differences between the first set of seed variables (seed mass 1 and germination rate 1 ). In the first set, it is the seeds that are either outcrossed or self-fertilized; in the latter, all seeds are produced by open pollination in the common garden trial, but by either selfed or outcrossed mother plants.

Late fitness traits: Number of flower stalks: After flowering and seed maturation, we harvested and counted all stalks from all plants in the common garden trial. Number of capsules: We counted the total number of capsules per individual plant. Survival rate: We sowed between 8 and 12 randomly chosen seeds from each of the harvested plants into $14 \mathrm{~cm}$ pots. The survival rate of germinated plants was assessed after 13 weeks. The pots were lightly irrigated every other week during this period. W1: We estimated the multiplicative fitness as $W 1=\#$ capsules $\times$ germination rate 2 . $W 2$ : This is the multiplicative fitness estimated as $W 2=W 1 \times$ seedling survival rate.

\section{Assessment of ID}

ID was estimated using a family based estimate for all paternal families (cf. Johnston and Schoen, 1994; Lynch and Walsh, 1998; Hansen, 2003):

$$
\delta_{i k l}(z)=1-\frac{z_{i s k l}}{\bar{z}_{i w k}}\left[\frac{1}{1+\hat{\sigma}^{2}\left(\bar{z}_{i w k}\right) / \bar{z}_{i w k}^{2}}\right]
$$

where $i$ denotes population identity (Small, Large), $j$ denotes cross type (outcrossed or selfed), $k=\{1,2, \cdot$. . , 9\} denotes paternal parent from the $P$-generation and $l=\{1,2, \cdot . \cdot, 9\}$ denotes maternal parent from the $P$-generation nested within populations (the same set of mothers were used for all crosses within each of the two populations), $z_{i s k l}$ denotes the phenotypic value of the iskl'th selfed second-generation offspring, $z_{i w k}$ is the paternal mean of the outcrossed first-generation offspring and, finally, $\hat{\sigma}^{2}\left(\bar{z}_{i w k}\right)$ is the variance of the mean $z$ within the outcrossed offspring from the $i k^{\prime}$ th paternal parent. The term in the square brackets multiplied onto $\frac{z_{i k k l}}{\bar{z} \text { ink }}$ removes the bias (to second order accuracy) introduced when estimating a ratio.

The reason to use the paternal parent mean of the outcrossed type in the denominator rather than merely the population average is to remove variation in $\delta_{i j k}(w)$ generated by variation among families per se (Kelly, 2005). Consider the situation in which offspring from some families perform better than offspring from others more or less regardless of cross type. If we then were to use the population mean rather than the family mean, we would detect (genetic) variation in $\delta_{i j k}(w)$ attributable to the general differences in offspring performance between families. But instead, we want to estimate whether selffertilized offspring from some families suffers more (or less) from being inbred compared with their outbred family than other self-fertilized families do (Johnston and Schoen, 1994).

\section{Statistical analyses and interpretation of parameters}

Population mean ID: For graphical presentation, we calculated the population mean inbreeding and associated 95\% confidence intervals (bias-corrected accelerated bootstrap, $\mathrm{BCa}$; Shao and $\mathrm{Tu}$, 1995) for all traits. Each confidence interval was based on re-sampling individuals within populations 5000 times.

Population differences and among-family variances: We analysed the inbreeding-depression measures $\left(\delta_{i k l}(z)\right)$ using linear mixed modelling. For all phenotypic traits considered, we analysed the model

$$
\delta_{i k l}(z)=\alpha_{i}+u_{k}+u_{l}+\varepsilon_{i k l}
$$

where $\alpha_{i}$ is the fixed effect of population identity, $u_{k}$ and $u_{l}$ are the random effects of paternal and maternal identity (from the $P$-generation), respectively, and finally, $\varepsilon_{i k l}$ is the random error unaccounted for by the three preceding terms. The two random terms, $u_{k}$ and $u_{l}$, are assumed to be normally distributed with zero means and variances $\sigma_{\mathrm{p}}^{2}$ and $\sigma_{\mathrm{m}}^{2}$, where the indices denote paternal and maternal identity, respectively. If one or both of these terms are statistically significant, that indicates that there is genetic variation in ID among families. We used SAS proc mixed with the REML option to estimate and test the random part of the model and the ML to estimate and test the fixed effect of population in the model.

Multivariate population differences: A more powerful way to assess population differences is to use the information from all traits simultaneously in a multivariate analysis of variance. Hence, we performed a multivariate analysis of variance on (a) the floral traits, (b) the fitness traits and (c) all 14 traits. The multivariate analysis of variance model had population identity as a fixed effect and maternal and paternal identity as random effects. We included the random effects merely to control their variation rather than wanting to estimate their effects. To test the population effect, we used the Pillai's Trace statistic (Jobson, 1996) and its significance was assessed by a randomization test in which we 
randomly assigned whole paternal sibships to populations 1000 times. These analyses were carried out using SAS proc glm.

Differences between floral and fitness traits: To test whether inbreeding affects floral traits as a whole differently than it does fitness traits, we compared the mean ID of the five floral traits with the mean ID of the nine fitness traits for all selfed individuals in the common garden trial. Consider the 14 traits indexed by $z_{t}$ $(t=1,2,3, \cdot \cdot \cdot, 14)$, so that $\delta_{i k l}\left(z_{t}\right)$ is the ID of the $z t^{\prime}$ th trait in the $i k l^{\prime}$ th selfed individual. The following statistic was evaluated to test whether inbreeding affects the two types of traits differently:

$$
y_{i k l}=\sum_{t \in \text { fitness }} \delta_{i k l}\left(\mathbf{z}_{t}\right) / 9-\sum_{t \in \text { floral }} \delta_{i k l}\left(\mathbf{z}_{t}\right) / 5
$$

The significance of the differences in Equation (3) was tested with one-sample $t$-tests for each of the two populations in which the $P$-values were estimated by a non-parametric bootstrap (Shao and Tu, 1995) using 1000 re-samples. The bootstrap tests were implemented in $R$ using the boot library (Canty and Ripley, 2002). We estimated $\mathrm{BCa}$ confidence intervals for the mean differences between floral and fitness traits based on 5000 re-samples.

To compare the paternal variances between the floral and the fitness trait sets, we calculated $\psi(z)=\hat{\sigma}_{p}^{2}(z) / \hat{\sigma}_{\varepsilon}^{2}(z)$ for each trait and compared the average of $\psi(z)$ $\left(\psi_{\text {floral }}-\psi_{\text {fitness }}\right)$. The significance of the difference was assessed based on a randomization test of 2000 replicates.

Genetic correlations: To test whether the ID in different traits is influenced by the same loci, we correlated the paternal mean ID among the different traits. We assessed the statistical significance of the correlations by randomization tests performed in $\mathrm{R}$ using the boot library (Canty and Ripley, 2002).

\section{Results}

Population and life-cycle differences in ID

Population means and the variation of the phenotypic traits measured in the common garden trial are presented in Table 1. Both populations exhibited significant mean ID in all floral traits (except for ovary dry weight in the Large population) ranging from 0.12 to 0.2 (Figure 1). Late fitness traits suffered even higher ID ranging from 0.44 to 0.74 . In contrast, seed traits hardly showed any significant ID.

In both populations, ID was significantly larger in fitness traits than in floral traits (Small: $t=2.71, \mathrm{df}=75$, $P=0.0084$; Large: $t=5.50, \mathrm{df}=72, P=0.0004)$. The mean differences ( $y_{i k l}$, see Equation (3)) were 0.088 in Small (95\% BCa confidence interval: $0.024 ; 0.149)$ and 0.239 in Large (0.159; 0.326).

There were hardly any significant differences in ID between the two populations, except for the germination rate 2 (Table 2). In the multivariate analyses, the populations did not differ in floral traits (Pillai's Trace $F_{5 ; 12}=1.88 ; P=0.1650$ ) or all traits combined (Pillai's Trace $\left.F_{14 ; 5}=2.07 ; P=0.2060\right)$. However, the Small population had a somewhat lower ID for the nine fitness traits combined (almost significant difference in Pillai's Trace,
Table 1 Population means and variation of the phenotypical values of outcrossed plants and selfed offspring for traits with significant inbreeding depression

\begin{tabular}{|c|c|c|c|c|}
\hline \multirow[t]{3}{*}{ Character } & \multicolumn{4}{|c|}{ Mean values } \\
\hline & \multicolumn{2}{|c|}{ Small population } & \multicolumn{2}{|c|}{ Large population } \\
\hline & Outcrossed & Selfed & Outcrossed & Selfed \\
\hline Sepal & 2.2 & 1.8 & 2.3 & 2.1 \\
\hline Petal & 3.3 & 2.8 & 3.0 & 2.6 \\
\hline Ovary & 0.7 & 0.7 & 0.7 & 0.8 \\
\hline Stamen & 1.8 & 1.4 & 1.8 & 1.4 \\
\hline Flower & 8.4 & 7.0 & 8.2 & 7.2 \\
\hline Stalks & 67.8 & 43.9 & 53.5 & 30.8 \\
\hline Capsules & 985.3 & 560.6 & 646.3 & 325.8 \\
\hline Survival & 0.7 & 0.4 & 0.5 & 0.3 \\
\hline W1 & 924.8 & 532.3 & 594.9 & 297.8 \\
\hline \multirow[t]{2}{*}{ W2 } & 690.9 & 268.7 & 370.0 & 143.2 \\
\hline & \multicolumn{4}{|c|}{$\begin{array}{l}\text { Coefficient of variation } \\
\text { (bootstrap s.e.) }\end{array}$} \\
\hline Sepal & $15.9 \%(1.7 \%)$ & $24.5 \%(1.9 \%)$ & $17.6 \%(2.0 \%)$ & $30.5 \%(2.7 \%)$ \\
\hline Petal & $17.8 \%(2.0 \%)$ & $26.3 \%(2.4 \%)$ & $16.9 \%(1.7 \%)$ & $27.8 \%(2.2 \%)$ \\
\hline Ovary & $22.0 \%(2.8 \%)$ & $37.6 \%(3.9 \%)$ & $23.6 \%(3.0 \%)$ & $65.6 \%(9.4 \%)$ \\
\hline Stamen & $13.5 \%(2.1 \%)$ & $38.3 \%(5.9 \%)$ & $13.3 \%(1.6 \%)$ & $43.9 \%(6.2 \%)$ \\
\hline Flower & $13.3 \%(1.5 \%)$ & $21.7 \%(2.1 \%)$ & $13.5 \%(1.4 \%)$ & $24.1 \%(1.9 \%)$ \\
\hline Stalks & $42.0 \%(6.5 \%)$ & $51.1 \%(5.0 \%)$ & $46.9 \%(5.8 \%)$ & $68.2 \%(5.5 \%)$ \\
\hline Capsules & $48.0 \%(7.8 \%)$ & $58.8 \%(5.5 \%)$ & $51.4 \%(6.4 \%)$ & $75.9 \%(6.4 \%)$ \\
\hline Survival & $32.9 \%(6.1 \%)$ & $60.8 \%(6.7 \%)$ & $47.9 \%(7.5 \%)$ & $93.7 \%(9.9 \%)$ \\
\hline W1 & $50.2 \%(7.9 \%)$ & $56.4 \%(5.3 \%)$ & $53.2 \%(6.2 \%)$ & $73.9 \%(6.8 \%)$ \\
\hline W2 & $54.1 \%(7.6 \%)$ & $89.1 \%(8.6 \%)$ & $81.7 \%(9.4 \%)$ & $127.8 \%(13.5 \%)$ \\
\hline
\end{tabular}

$\left.F_{9 ; 10}=2.95 ; P=0.0665\right)$. For one subset of fitness traitscapsule number, seed mass 2, germination rate 2 and survival rate-we found a marginally significant lower ID in the Small population $(P=0.0438)$, but for other combinations, the $P$-values exceeded $5 \%$.

\section{Among-family variance in ID}

The paternal variances in ID were higher than the corresponding maternal variances (Table 2), which is in part an artefact because of the way the individual ID was estimated, using the crossbred paternal mean in the denominator in Equation (1). The residual variances (rightmost column in Table 2) were substantially higher than the genetic variances for all the considered traits. Nonetheless, we found significant paternal genetic variance of ID in all floral traits, except for stamens, and in all seed-related fitness traits (seed mass 1 and 2, germination rate 1 and 2), but not in the late fitness traits (Table 2). The paternal variance in ID was higher in the set of floral traits compared with fitness $\left(\psi_{\text {floral }}-\psi_{\text {fitness }}=0.269, t=3.44, \mathrm{df}=12, P=0.0130\right)$. The distribution of paternal-family means of ID is shown in Figure 2. The family-level variance and mean of ID were negatively correlated $(r=-0.18, P=0.0041)$.

\section{Genetic correlations of ID}

ID of floral traits was positively inter-correlated (Table 3), with the exception of stamens that were negatively correlated with ovaries. The late fitness traits were also positively inter-correlated. Among early fitness traits, we found a positive correlation between seed mass and germination in the seeds harvested from the tested crosses (seed mass 2, germination 2). There were no 
significant correlations between floral and fitness traits. Among early and late fitness traits, seed mass 1 and germination rate 2 were correlated with multiplicative fitness (W1).

\section{Discussion}

The magnitude of ID in $S$. nutans

The ID found in the late fitness traits of $S$. nutans, that is the number of stalks and capsules, survival and multiplicative fitness, is very high compared with other studies and other species. In addition, other studies have found selfing disadvantages of $S$. nutans in traits such as pollen-tube growth and zygote survival $(\delta=0.06-0.08$; Hauser and Siegismund, 2000). By comparison, Husband and Schemske (1996, their Figure 3 report species' average ID in fecundity and growth to range between 0.28 and 0.33 for outcrossed species.

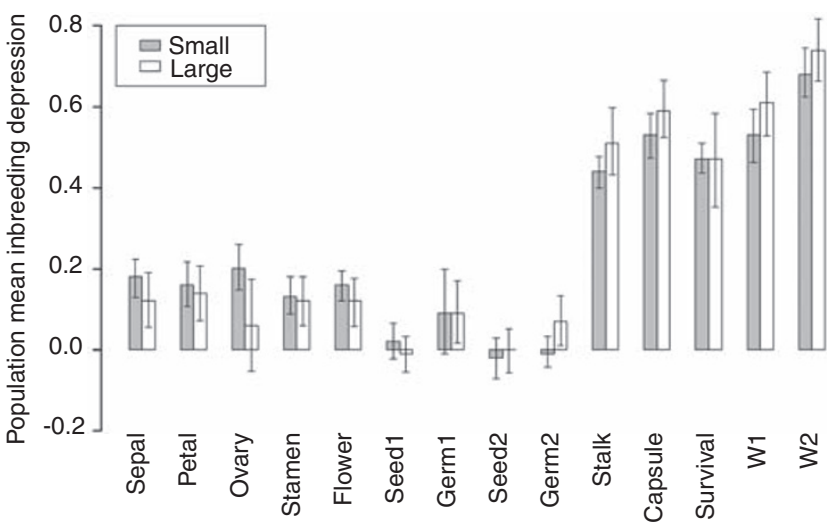

Figure 1 Population mean ID in floral and fitness traits in two populations of S. nutans (Small, Large). Bars represents $95 \% \mathrm{BCa}$ confidence intervals. Traits with confidence intervals not containing zero are significantly different from zero.
Yet, for other Caryophyllaceae species, Husband and Schemske's appendix actually points towards similarly high levels of cumulative ID (mean $\delta$ between 0.50 and 0.83). Recently, high cumulative ID has been reported for the close Silene-relatives Lychnis flos-cuculi (0.57-0.87: Hauser and Loeschcke, 1994, 1995; 0.91 under drought stress: Hauser and Loeschcke, 1996) and Lychnis viscaria (up to 0.629; Mustajärvi et al., 2005), but also for species from other plant families, for example the lily Bulbine bulbosa (0.85; Owen et al., 2007) or Magnolia obovata (up to 0.69/0.97; Ishida, 2006).

\section{Populations size and ID}

The two populations considered here both have intermediate selfing rates (Weidema and Philipp, unpublished) and have probably persisted under constant conditions for a long period of time, but their sizes differ orders of magnitude. Regardless hereof little differences in ID were found between the two populations. However, although mostly not significant, there was a consistent trend in the multivariate analyses of fitness traits that the Small population suffered less from ID. This may give grounds to presume that there was some degree of purging in the Small population, but that the effect was too small compared with the within-population variation of ID to be detectable from a sample of 9 paternal and 9 maternal plants ( 81 crosses) per population. In the end, the evidence for purging remains inconclusive in this study.

There seems to be no consistent relationship between population size and ID in general (Byers and Waller, 1999). There are several possible explanations for populations size not to be associated with the level of ID and purging. First, population size may be a bad predictor of the level of inbreeding (Leimu et al., 2006; Honnay and Jacquemyn, 2007). If dispersal of pollen and seeds is limited, then even large outcrossing populations may be genetically structured and show some degree of inbreeding because of matings of related plants (Byers

Table 2 Linear mixed-effects analyses of inbreeding depression coefficients $(\delta)$ according to Equation (2)

\begin{tabular}{|c|c|c|c|c|c|c|c|c|c|}
\hline \multirow[t]{2}{*}{ Character } & \multicolumn{4}{|c|}{ Population means } & \multicolumn{5}{|c|}{ Population variances } \\
\hline & Small & Diff. & s.e. (Diff.) & $\mathrm{t}$-value & $\sigma_{p}^{2}$ & Q-value & $\sigma_{m}^{2}$ & Q-value & $\sigma_{\varepsilon}^{2}$ \\
\hline \multicolumn{10}{|l|}{ Floral } \\
\hline Sepal & 0.184 & 0.057 & 0.077 & 0.74 & 0.015 & $16.82^{* * *}$ & 0.010 & $9.54^{* * *}$ & 0.036 \\
\hline Petal & 0.166 & 0.031 & 0.070 & 0.45 & 0.018 & $20.44^{* * *}$ & 0.002 & 0.56 & 0.038 \\
\hline Ovary & 0.199 & 0.136 & 0.094 & 1.44 & 0.035 & $22.69^{* * *}$ & 0.001 & 0.02 & 0.068 \\
\hline Stamen & 0.131 & 0.012 & 0.033 & 0.36 & 0.002 & 2.25 & 0.000 & 0.11 & 0.023 \\
\hline Flower & 0.162 & 0.046 & 0.059 & 0.78 & 0.012 & $17.97^{* * *}$ & 0.003 & 2.07 & 0.026 \\
\hline \multicolumn{10}{|l|}{ Fitness } \\
\hline Seed mass 1 & 0.020 & 0.029 & 0.037 & 0.78 & 0.004 & $7.97^{* *}$ & 0.001 & 0.77 & 0.018 \\
\hline Germination rate 1 & 0.085 & -0.004 & 0.109 & 0.04 & 0.035 & $14.80^{* * *}$ & 0.013 & $4.06^{* * *}$ & 0.091 \\
\hline Seed mass 2 & -0.016 & -0.014 & 0.044 & 0.33 & 0.005 & $4.92^{*}$ & 0.000 & 0.00 & 0.032 \\
\hline Germination rate 2 & -0.004 & -0.076 & 0.032 & 2.35 & 0.003 & $3.93^{*}$ & 0.000 & 0.00 & 0.018 \\
\hline Stalks $\#$ & 0.436 & -0.079 & 0.055 & 1.42 & 0.000 & 0.00 & 0.000 & 0.00 & 0.094 \\
\hline Capsules $^{\#}$ & 0.526 & -0.069 & 0.051 & 1.35 & 0.000 & 0.00 & 0.000 & 0.00 & 0.080 \\
\hline Survival rate & 0.472 & 0.005 & 0.075 & 0.07 & 0.007 & 0.56 & 0.000 & 0.00 & 0.140 \\
\hline W1 & 0.526 & -0.082 & 0.051 & 1.60 & 0.003 & 0.33 & 0.000 & 0.00 & 0.070 \\
\hline W2 & 0.686 & -0.054 & 0.056 & 0.96 & 0.003 & 0.34 & 0.000 & 0.00 & 0.085 \\
\hline
\end{tabular}

The 'Small' column gives the population mean ID in the Small population. The 'Diff.' is the difference between the mean $\delta$ in the Small population minus that of the Large population, and 's.e. (Diff)' is the standard error hereof. The symbols $\sigma_{\mathrm{p}}^{2}, \sigma_{\mathrm{m}}^{2}$ and $\sigma_{\mathrm{a}}^{2}$ are paternal, maternal and residual variances, respectively. The levels of statistical significance are ${ }^{* * *} P<0.001 ;{ }^{* * P}<0.01 ;{ }^{*} P<0.05$. 


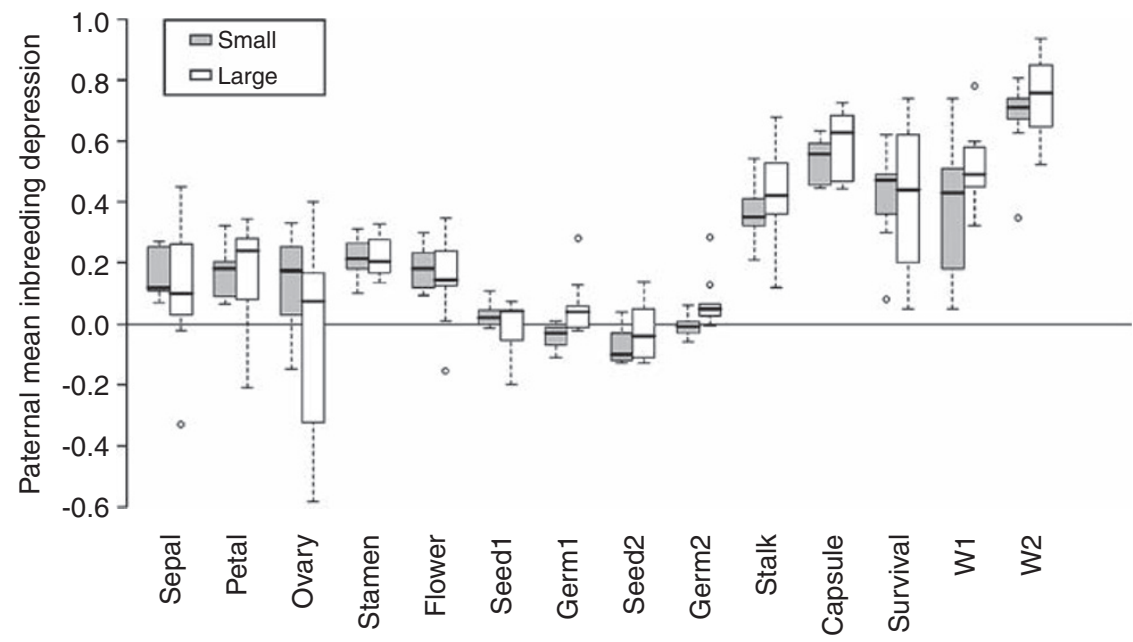

Figure 2 Distribution of paternal-family means of ID ( $\ddot{a}$, see formula 1) for floral and fitness traits within two populations of S. nutans (Small, Large). Boxes indicate median and interquartile range; whiskers indicate the most extreme values within 1.5 times the interquartile range; values more extreme are indicated by circles.

Table 3 Pair-wise correlations of paternal mean inbreeding depression $(\delta)$ among all traits

\begin{tabular}{|c|c|c|c|c|c|c|c|c|c|c|c|c|c|c|c|c|}
\hline Character & Sepals & Petals & Ovary & Stamen & Flower & Seed1 & Germ1 & Seed 2 & Germ2 & Stalks & Capsules & Survived & W1 & W2 & Fitness & Floral \\
\hline pals & & 0.82 & 0.86 & -0.36 & 0.94 & 0.10 & 0.29 & 0.10 & -0.16 & -0.11 & -0.36 & -0.42 & -0.30 & -0.28 & -0.24 & 0.95 \\
\hline Petals & 0.001 & & 0.77 & -0.19 & 0.94 & 0.15 & 0.36 & 0.14 & -0.12 & -0.20 & -0.30 & -0.43 & -0.23 & -0.31 & -0.14 & 0.91 \\
\hline Ovary & 0.001 & 0.003 & & -0.58 & 0.89 & 0.15 & 0.16 & 0.29 & -0.12 & -0.17 & -0.36 & -0.27 & -0.33 & -0.24 & -0.29 & 0.95 \\
\hline Stamen & 0.133 & 0.452 & 0.010 & & -0.28 & 0.23 & -0.19 & -0.33 & 0.05 & -0.01 & 0.16 & -0.03 & 0.24 & -0.12 & 0.08 & -0.37 \\
\hline Flower & 0.001 & 0.001 & 0.001 & 0.275 & & 0.14 & 0.28 & 0.15 & -0.14 & -0.18 & -0.37 & -0.42 & -0.29 & -0.33 & -0.22 & 0.98 \\
\hline Seed1 & 0.640 & 0.543 & 0.551 & 0.365 & 0.557 & & -0.18 & 0.03 & 0.06 & 0.35 & 0.37 & 0.43 & 0.49 & 0.33 & 0.40 & 0.17 \\
\hline Germ1 & 0.244 & 0.142 & 0.532 & 0.448 & 0.243 & 0.447 & & -0.31 & -0.34 & -0.05 & -0.11 & -0.13 & -0.16 & 0.10 & 0.21 & 0.24 \\
\hline Seed2 & 0.715 & 0.608 & 0.273 & 0.185 & 0.568 & 0.926 & 0.215 & & 0.64 & -0.11 & -0.22 & -0.11 & -0.16 & -0.23 & 0.04 & 0.19 \\
\hline Germ2 & 0.483 & 0.633 & 0.634 & 0.852 & 0.551 & 0.787 & 0.183 & 0.005 & & 0.42 & 0.44 & 0.29 & 0.46 & 0.18 & 0.47 & -0.14 \\
\hline Stalks & 0.662 & 0.417 & 0.472 & 0.976 & 0.464 & 0.158 & 0.841 & 0.624 & 0.089 & & 0.82 & 0.53 & 0.83 & 0.61 & 0.67 & -0.18 \\
\hline Capsules & 0.149 & 0.235 & 0.149 & 0.552 & 0.141 & 0.138 & 0.674 & 0.365 & 0.059 & 0.001 & & 0.79 & 0.93 & 0.80 & 0.74 & -0.37 \\
\hline Survival & 0.083 & 0.090 & 0.283 & 0.916 & 0.094 & 0.067 & 0.623 & 0.668 & 0.245 & 0.021 & 0.001 & & 0.77 & 0.91 & 0.65 & -0.39 \\
\hline W1 & 0.233 & 0.360 & 0.192 & 0.329 & 0.241 & 0.037 & 0.553 & 0.510 & 0.048 & 0.001 & 0.001 & 0.001 & & 0.76 & 0.73 & -0.31 \\
\hline W2 & 0.260 & 0.183 & 0.332 & 0.653 & 0.176 & 0.168 & 0.694 & 0.363 & 0.435 & 0.007 & 0.001 & 0.001 & 0.001 & & 0.72 & -0.31 \\
\hline Fitness & 0.364 & 0.584 & 0.250 & 0.766 & 0.389 & 0.097 & 0.408 & 0.876 & 0.047 & 0.004 & 0.001 & 0.005 & 0.002 & 0.002 & & -0.26 \\
\hline Floral & 0.001 & 0.001 & 0.001 & 0.145 & 0.001 & 0.443 & 0.320 & 0.457 & 0.517 & 0.475 & 0.134 & 0.124 & 0.229 & 0.205 & 0.305 & \\
\hline
\end{tabular}

Correlation coefficients are listed above the diagonal and their associated test probabilities (randomization tests) are listed below the diagonal. Bold face written correlations are significant with $P<0.05$. Note, however, that the tests presented here are liberal because there is no correction for multiple testing. The 'Fitness' and the 'Floral' traits are the mean $\delta$ in the fitness and the floral traits, respectively, calculated according to Equation (3).

and Waller, 1999). Further, gene flow among populations through pollen and seed dispersal may mitigate genetic effects of small, fragmented populations (Kramer et al., 2008 ) and render purging processes void. For example, Zealand populations of S. nutans are not very isolated from one another in that the multi-locus Fst value is 0.139 (s.e. $=0.036$; Weidema and Philipp, unpublished). Moreover, population sizes and inbreeding may have fluctuated in the past, or a population may have adapted to local environmental conditions, so that the particular history of a population may override current processes of inbreeding and purging (Mills, 2007; Leimu et al., 2008), although we suppose the populations studied here to have been constant in size and inbreeding level over a long period of time.

Second, regarding the genetic basis of ID, purging is expected to happen, if ID is caused by recessive deleterious alleles that are exposed to selection when homozygous, whereas purging is not possible if ID is due to overdominance (Allendorf and Luikart, 2007; Wright et al., 2008). But even deleterious recessive alleles may be difficult to purge, if they have comparatively high dominance coefficients and if their effects are only mildly deleterious (Charlesworth and Charlesworth, 1999; Wang et al., 1999; Allendorf and Luikart, 2007). Hence, if ID is based on mildly deleterious recessives or overdominance, which also can occur together, purging effects may be low and difficult to detect (Crnokrak and Barrett, 2002). In addition, purging is less likely to happen for plants with perennial life histories (Byers and Waller, 1999) such as S. nutans, and generally is less effective for larger plants (Scofield and Schultz, 2006).

Altogether, the slight tendency of the Small population to suffer less from ID would be consistent with the notion that ID is caused by a mixed system with many deleterious recessive alleles of small effect and few with 
large effect, and possibly with some overdominance, so that only a minor part of the genetic load, that is lethal or sub-lethal mutations, can be purged (Byers and Waller, 1999; Charlesworth and Charlesworth, 1999; Crnokrak and Barrett, 2002).

\section{Differences between floral and fitness traits}

Most studies on ID have focussed on life-history traits related to fitness, and only relatively few have considered morphological traits, such as floral traits. In these, there seems to be a trend that morphological traits suffer less from inbreeding than fitness (DeRose and Roff, 1999 but see; Ellmer and Andersson, 2004; Wright et al., 2008).

In our populations of $S$. nutans, ID was significantly lower for floral traits than for (late) fitness traits. A simple explanation could be that selection acts more strongly on flowers than on fitness, which does, however, not seem reasonable. Alternatively, selection acting on floral traits may be stabilizing, in contrast to selection on fitness traits that likely is directional. Genetic variation may thus become lower for the floral traits. Larger floral display probably increases male and female fitness, as in many other species (Philipp and Hansen, 2000; Ashman and Diefenderfer, 2001), but may also give more infection by anther smut fungi (Ustilago violacea; Shykoff et al., 1997). The $S$. nutans populations we studied are commonly infected by anther smut fungi (Hansen, personal observation, 2001). Stabilizing selection on corolla size and through genetic correlations (cf. Table 3) on other floral traits, might, therefore, operate in S. nutans. Coefficients of variation are hence substantially smaller for the floral traits than for the five fitness traits suffering strong ID (number of stalks and capsules, survival rate and the two multiplicative fitness estimates) (Table 1). This could be the result of stronger developmental canalization in the floral traits (Schlichting and Pigliucci, 1998). An alternative explanation could be that the floral traits have lower levels of directional dominance variance than the fitness traits (DeRose and Roff, 1999).

\section{Differences between early and late fitness traits}

The early fitness traits, seed mass and germination rate, were much less affected by ID than the late fitness traits. This could be a result of genes that are expressed early and have a cumulative pleiotropic effects on genes expressed later on (Husband and Schemske, 1996). However, this is not likely here, as early and late fitness traits seem to be genetically decoupled, as indicated by the lack of correlation of paternal mean ID for these groups of fitness traits (Table 3 ).

A more likely explanation is that early ID is caused by (sub-)lethal alleles that are purged quite effectively, whereas mildly deleterious (nearly) additive alleles that are difficult to purge are responsible for ID suffered in later life stages. Consequently, differences in ID between inbred (small) and outcrossed (large) populations will be prevalently found in early traits, but not in late traits (Goodwillie and Knight, 2006; Ishida, 2008). This explanation is in accord with the findings of Husband and Schemske's (1996) review that an increase of ID from low to high levels during the life cycle is typical for selfing plants, whereas outcrossing plants tend to suffer equally strong in early and late traits. With the mixed mating system of $S$. nutans, it seems that purging of (sub-) lethal alleles affecting seed traits is effective regardless of population size.

\section{Genetic variation and mating-system evolution}

We found significant variance in ID among paternal families for floral traits (with the exception of stamens) and seed-related fitness traits, whereas variance estimates were not significant for late fitness traits (Table 2). Apparently, there is an association between high ID and low genetic variation therein and vice versa. However, if we look at Figure 2, family-mean ID for late fitness traits was strongly variable, with an almost threefold difference between the lowest and highest values for multiplicative fitness W2. In an additional analysis, in which paternal families were treated as fixed effects, many significant pair-wise differences between paternal families were found for the fitness traits. Some families thus do have higher (or lower) ID than others, despite the lack of significant variation in ID in the tests of our main statistical model (2).

Many other studies on this topic have found some genetic variation in ID, often reported as an interaction between family groups and 'pollination treatment' (for example Mutikainen and Delph, 1998; Jóhannsson et al., 1998, Fishman, 2001; Picó et al., 2004; Oakley and Winn, 2008). However, in most experiments such family variation includes a component because of differences in fitness among outcrossed offspring fertilized by different sets of pollen donors (Fox, 2005; Kelly, 2005). This bias makes it difficult to infer the existence or magnitude of genetic variation from family variation. In our study, we used a fully factorial crossing design (North Carolina II), that is all outcrossed paternal half-sib families included the same set of maternal plants, which minimizes the bias described above.

The most exciting question about genetic variation in ID is whether it may change the conditions under which different types of mating systems are evolutionary stable (Uyenoyama et al., 1993). More specifically, genetic variation in ID relaxes the conditions for the spread of selfing-modifier genes, so that even with mean ID above 0.5 selfing-modifier genes may still spread. Hence, family-level ID may be more important for matingsystem evolution than population-level ID (Kelly and Tourtellot, 2006).

At the population level, the high $(\delta>0.5)$ ID of multiplicative fitness observed in this study (Figure 1) suggests that the mixed mating system of $S$. nutans is not stable, but should evolve towards outcrossing (Goodwillie et al., 2005). However, for all fitness parameters, including multiplicative fitness, there were families with ID below 0.5. For the cumulative fitness measure $W 2$, which showed the highest average ID $(\delta=0.74)$, there was a probability of approximately $5 \%$ for families to have ID lower than 0.5. Hence, complete outcrossing may be prevented by recurring evolution of prevalently selfing lines that suffer only moderately from ID, whereas evolution towards increased selfing will be prevented by the generally high ID in late fitness traits that apparently is difficult to purge. Thus, the mixed mating system of $S$. nutans may have been evolutionary stable over a long period of time. 


\section{Acknowledgements}

For discussions and comments, we thank Ole K Hansen, Lise B Hansen and Marianne Philipp. The staffs at the Botanical Garden in Copenhagen and at the University of Copenhagen's field station facility at Taastrup are thanked for maintaining the plants used in the common garden trial. Furthermore, we thank Ruth B Jacobsen, Karna Heinsen and Karina L Sørensen for helping out with the practical work.

\section{References}

Allendorf FW, Luikart G (2007). Conservation and the Genetics of Populations. Blackwell: Oxford.

Ashman TL, Diefenderfer C (2001). Sex ratio represents a unique context for selection on attractive traits: consequences for the evolution of sexual dimorphism. Am Nat 157: 334-347.

Bruun HH, Fritzbøger B, Rindel PO, Hansen UL (2001). Plant species richness in grasslands: the relative importance of contemporary environment and land-use history since the Iron Age. Ecography 24: 569-578.

Byers DL, Waller DM (1999). Do plant populations purge their genetic load? Effects of population size and mating history on inbreeding depression. Annu Rev Ecol Syst 30: 479-513.

Canty A, Ripley B (2002). The boot library. Retrievable at http:// mirrors.-sunsite.dk/cran/with documentation at the same URL (by May 2002).

Charlesworth D (2006). Evolution of plant breeding systems. Curr Biol 16: R726-R735.

Charlesworth B, Charlesworth D (1999). The genetic basis of inbreeding depression. Genet Res Camb 74: 329-340.

Charlesworth D, Morgan MT, Charlesworth B (1990). Inbreeding depression, genetic load, and the evolution of outcrossing rates in a multilocus system with no linkage. Evolution 44: 1469-1489.

Crnokrak P, Barrett SCH (2002). Purging the genetic load: a review of the experimental evidence. Evolution 56: 2347-2358

DeRose MA, Roff DA (1999). A comparison of inbreeding depression in life-history and morphological traits in animals. Evolution 53: 1288-1292.

Ellmer M, Andersson S (2004). Inbreeding depression in Nigella degenii (Ranunculaceae): fitness components compared with morphological and phenological characters. Int J Plant Sci 165: 1055-1061.

Ellstrand NC, Elam DR (1993). Population genetic consequences of small population size: implications for plant conservation. Annu Rev Ecol Syst 24: 217-242.

Fishman L (2001). Inbreeding depression in two populations of Arenaria uniflora (Caryophyllaceae) with contrasting mating systems. Heredity 86: 184-194.

Fox CW (2005). Problems in measuring among-family variation in inbreeding depression. Am J Bot 92: 1929-1932.

Goodwillie C, Knight MC (2006). Inbreeding depression and mixed mating in Leptosiphon jepsonii: a comparison of three populations. Ann Bot 98: 351-360.

Goodwillie C, Kalisz S, Eckert CG (2005). The evolutionary enigma of mixed mating systems in plants: occurrence, theoretical explanations, and empirical evidence. Annu Rev Ecol Evol Syst 36: 47-79.

Hansen T (2003). Quantitative genetics of sexual allocation and inbreeding depression in hermaphroditic plants with special reference to Silene nutans (Caryophyllaceae). PhD Thesis, University of Copenhagen.

Hauser TP, Loeschcke V (1994). Inbreeding depression and mating-distant dependent offspring fitness in large and small populations of Lychnis flos-cuculi (Caryophyllaceae). J Evol Biol 7: 609-622.
Hauser TP, Loeschcke V (1995). Inbreeding depression in Lychnis flos-cuculi (Caryophyllaceae): effects of different levels of inbreeding. J Evol Biol 8: 589-600.

Hauser TP, Loeschcke V (1996). Drought stress and inbreeding depression in Lychnis flos-cuculi (Caryophyllaceae). Evolution 50: 1119-1126.

Hauser TP, Siegismund HR (2000). Inbreeding and outbreeding effects on pollen fitness and zygote survival in Silene nutans (Caryophyllaceae). J Evol Biol 13: 446-454.

Hauser TP, Weidema IR (2000). Extreme variation in flowering time between populations of Silene nutans. Hereditas 132: 95-101.

Honnay O, Jacquemyn H (2007). Susceptibility of common and rare plant species to the genetic consequences of habitat fragmentation. Conserv Biol 21: 823-831.

Husband B, Schemske D (1996). Evolution of the magnitude and timing of inbreeding depression in plants. Evolution 50: 50-74.

Ishida K (2006). Maintenance of inbreeding depression in a highly self-fertilizing tree, Magnolia obovata Thunb. Evol Ecol 20: 173-191.

Ishida K (2008). Effects of inbreeding on the magnitude of inbreeding depression in a highly self-fertilizing tree, Magnolia obovata. Ecol Res 23: 995-1003.

Jobson JD (1996). Applied Multivariate Analysis. Springer Verlag: Berlin.

Jóhannsson MH, Gates MJ, Stephenson AG (1998). Inbreeding depression affects pollen performance in Cucurbita texana. J Evol Biol 11: 579-588.

Johnston MO, Schoen DJ (1994). On the measurement of inbreeding depression. Evolution 48: 1735-1741.

Jürgens A, Witt T, Gottsberger G (1996). Reproduction and pollination in central European populations of Silene and Saponaria species. Bot Acta 109: 316-324.

Kelly JK (2005). Family level inbreeding depression and the evolution of plant mating systems. New Phytol 165: 55-62.

Kelly JK, Tourtellot MK (2006). The genetic analysis of family structured inbreeding depression studies. Heredity 97: 346-354.

Kramer AT, Ison JL, Ashley MV, Howe HF (2008). The paradox of forest fragmentation genetics. Conserv Biol 22: 878-885.

Leimu R, Mutikainen P, Koricheva J, Fischer M (2006). How general are positive relationships between plant population size, fitness and genetic variation? J Ecol 94: 942-952.

Leimu R, Kloss L, Fischer M (2008). Effects of experimental inbreeding on herbivore resistance and plant fitness: the role of history of inbreeding, herbivory and abiotic factors. Ecol Lett 11: 1101-1110.

Lynch M, Walsh B (1998). Genetics and Analysis of Quantitative Traits. Sinauer Associates: Sunderland.

Mills LS (2007). Conservation of Wildlife Populations: Demography, Genetics, and Management. Blackwell Publishing: Oxford.

Mustajärvi K, Siikamäki P, kerberg A (2005). Inbreeding depression in perennial Lychnis viscaria (Caryophyllaceae): effects of population mating history and nutrient availability. Am J Bot 92: 1853-1861.

Mutikainen P, Delph LF (1998). Inbreeding depression in gynodioecious Lobelia siphilitica: among-family differences override between-morph differences. Evolution 52: 1572-1582.

Oakley CG, Winn AA (2008). Population-level and family-level inbreeding depression in a cleistogamous perennial. Int I Plant Sci 169: 523-530.

Ouborg NJ, Vergeer P, Mix C (2006). The rough edges of the conservation genetics paradigm for plants. J Ecol 94: 1233-1248.

Owen K, Vaughton G, Ramsey M (2007). Facilitated autogamy and costs of selfing in the perennial herb Bulbine bulbosa (Asphodelaceae). Int J Plant Sci 168: 579-585.

Philipp M, Hansen T (2000). The influence of plant and corolla size on pollen deposition and seed set in Geranium sanguineum (Geraniaceae). Nord J Bot 20: 129-140.

Picó FX, Ouborg NJ, Van Groenendael JM (2004). Evaluation of the extent of among-family variation in inbreeding depression in the perennial herb Scabiosa columbaria (Dipsacaceae). Am J Bot 91: 1183-1189. 
Schlichting CD, Pigliucci M (1998). Phenotypic Evolution A Reaction Norm Perspective. Sinauer Associates: Sunderland.

Scofield DG, Schultz ST (2006). Mitosis, stature and evolution of plant mating systems: low- $\Phi$ and high- $\Phi$ plants. Proc $R$ Soc B 273: $275-282$

Shao J, Tu D (1995). The Jackknife and Bootstrap. Springer Verlag: Berlin. Shykoff JA, Bucheli E, Kaltz O (1997). Anther smut disease in Dianthus silvester (Caryophyllaceae): natural selection on floral traits. Evolution 51: 383-392.

Uyenoyama MK, Waller DM (1991a). Coevolution of selffertilization and inbreeding depression. I. Mutation-selection balance at one and two loci. Theor Popul Biol 40: 14-46.

Uyenoyama MK, Waller DM (1991b). Coevolution of selffertilization and inbreeding depression. II. Symmetric overdominance in viability. Theor Popul Biol 40: 47-77.

Uyenoyama MK, Waller DM (1991c). Coevolution of selffertilization and inbreeding depression. III. Homo- zygous lethal mutations at multiple loci. Theor Popul Biol 40: $173-210$

Uyenoyama MK, Holsinger KE, Waller DM (1993). Ecological and genetic factors directing the evolution of self-fertilization. In: Futuyma D, Antonovics J (eds). Oxford Surveys in Evolutionary Biology, Vol. 9 Oxford University Press: Oxford, pp 327-381.

Van Rossum F, Prentice HC (2004). Structure of allozyme variation in Nordic Silene nutans (Caryophyllaceae): population size, geographical position and immigration history. Biol J Linn Soc 81: 357-371.

Wang J, Hill WG, Charlesworth D, Charlesworth B (1999). Dynamics of inbreeding depression due to deleterious mutations in small populations: mutation parameters and inbreeding rate. Genet Res Camb 74: 165-178.

Wright LI, Tregenza T, Hosken DJ (2008). Inbreeding, inbreeding depression and extinction. Conserv Genet 9: 833-843. 